\title{
Acculturation and Colorectal Cancer Screening Among Older Latino Adults: Differential Associations by National Origin
}

\author{
Aimee Afable-Munsuz, $P h D^{7}$, Su-Ying Liang, $P h D^{2}$, Ninez A. Ponce, MPP, $P h D^{3}$, \\ and Judith M. E. Walsh, MD, MPH ${ }^{4}$
}

'Division of General Internal Medicine, Department of Medicine, University of California, San Francisco, CA, USA; ${ }^{2}$ Deparment of Clinical Pharmacy, University of California, San Francisco, CA, USA; ${ }^{3}$ Department of Health Services, University of California, Los Angeles, CA, USA; ${ }^{4}$ Women's Health Clinical Research Center, University of California, San Francisco, CA, USA.

BACKGROUND: Although modest improvements in colorectal cancer (CRC) screening utilization have occurred, rates remain low among Latinos. It is unclear whether acculturation plays a role in the utilization of CRC screening.

OBJECTIVE: This study aimed to examine the relationships between acculturation and CRC screening among older Mexican, Puerto-Rican and Cuban adults.

DESIGN: Cross-sectional observational study.

SUBJECTS: Latinos 50 years and older, never diagnosed with CRC, and who were surveyed in the 2000, 2003 and 2005 National Health Interview Survey (NHIS).

MEASURES: We measured acculturation with US nativity and language of interview, and examined three different CRC screening outcomes: fecal occult blood test (FOBT) in the past year, up-to-date endoscopy and any up-to-date CRC screening. Logistic regression models were adjusted for predisposing, enabling and health-care need factors consistent with the behavioral model of health-care utilization.

MAIN RESULTS: In adjusted analyses, US nativity was positively associated with up-to-date endoscopy among Mexicans (OR: 1.5; 95\% CI: 1.1, 2.2), but negatively associated with FOBT in the past year among Puerto Ricans (OR: 0.3; 95\% CI: 0.2, 0.7). In contrast to this latter finding among Puerto Ricans, English language interview was positively associated with FOBT in the past year (OR: 2.5; 95\% CI: 1.1, 5.4).

CONCLUSION: Results underscore the importance of stratification by national origin in studies of acculturation and cancer screening and of targeting less acculturated adults to promote CRC screening. Clinicians, however, should consider the complexity of acculturation and treat US nativity and language preference as independent dimensions among their Latino patients.

KEY WORDS: colorectal cancer screening; Latinos; aculturation.

Received August 7, 2008

Revised February 27, 2009

Accepted May 6, 2009

Published online May 27, 2009
J Gen Intern Med 24(8):963-70

DOI: $10.1007 /$ s11606-009-1022-9

(C) Society of General Internal Medicine 2009

\section{INTRODUCTION}

Colorectal cancer is the second leading cause of cancer-related death in the US ${ }^{1}$. Colorectal cancer (CRC) screening has been shown to effectively reduce mortality and morbidity from colorectal cancer due to early detection and treatment of cancer or precancerous lesions and is recommended for all average risk adults aged 50 and over ${ }^{2}$. Modest improvements in the utilization of CRC screening procedures have occurred in the past few years in the context of expanding Medicare coverage $^{3}$. However, wide health disparities remain among Latino and non-Latino Whites, Asians and non-Latino Whites, and immigrants and their native counterparts ${ }^{4-8}$.

Immigrants' poorer cancer screening practices relative to US natives may be related in part to immigrants having different values and beliefs about health service use ${ }^{9-11}$ or low levels of acculturation to US social norms concerning cancer screening. Several studies among Latino and Asian ethnic minority groups examine acculturation's influence on colorectal cancer screening with mixed findings. Two studies on acculturation and CRC screening among Latinos found no association between acculturation and CRC screening after accounting for socio-economic factors and access to care ${ }^{12,13}$. In contrast, the majority of studies conducted in various Asian ethnic groups found that greater acculturation was associated with a higher likelihood of CRC screening ${ }^{14-19}$. Interpreting results of these studies is challenging due to varying study methodologies. Each study used a different measure of acculturation. Some used variants of English language preference or proficiency $^{12,16,17}$, or time in the US measures ${ }^{18,19}$, while others used composite measures of acculturation ${ }^{13-15}$ reflecting concepts of language preference, time and co-ethnic relationships. Two studies measured ever using any CRC screening $\operatorname{method}^{13,15}$, while the remaining studies measured CRC screening based on recommended guidelines [i.e., fecal occult blood test (FOBT) in the past year or endoscopy in the past 5 years] ${ }^{12,14,16-19}$. The majority of the studies relied on convenience samples of adults, with notable exceptions ${ }^{12,17,19}$.

Most importantly, among the studies focusing on Latinos, none stratified analyses by country of origin. Past research has shown differential relationships between acculturation and 
health by ethnic group ${ }^{20,21}$; thus, aggregate analyses may underestimate the influence of acculturation, mask important ethnic group-specific patterns or produce contradictory findings. For breast cancer screening, national studies lumping Latino groups together and those that have had a more regional focus have produced inconsistent results with regard to the associations between acculturation and breast cancer screening, possibly because of varying ethnic group distributions $^{22-27}$. Further, each ethnic group experiences a unique acculturation experience shaped by US immigration policy and the social and economic conditions of the receiving community at the time of immigration ${ }^{28,29}$.

\section{Study Objectives}

The primary goal of this study is to investigate the relationship between acculturation and colorectal cancer (CRC) screening in Latino adults 50 and older using nationally representative data. We address two important gaps in the literature. First, we stratify our analyses by national origin, examining whether the relationships between acculturation and CRC screening vary among groups of Mexican, Puerto-Rican and Cuban origin; no single existing study has done such a comparative analysis. Second, no past nationally representative study on acculturation and CRC screening has examined the different CRC screening modalities separately, an important consideration given the variability in access to, cost and nature of the different modalities. We examine the relationship between acculturation and three different CRC screening outcomes separately-FOBT, endoscopy and any CRC screening modality-all according to recommended guidelines. Acculturation has been conceptualized as a multidimensional process whereby immigrants adopt selected values and behaviors of their host culture and may discard or retain those of their country of origin ${ }^{28,30-32}$. We relate acculturation to $\mathrm{CRC}$ screening taking into consideration factors central to the behavioral model of health-care utilization, which suggests that health service utilization is a function of individuals' predisposition due to age, gender or social status, factors that enable or impede use of services (such as health insurance), and actual need for health care ${ }^{33}$.

\section{METHODS}

This study used data from the 2000, 2003 and 2005 National Health Interview Survey (NHIS), the principal source of information on the health of the civilian non-institutionalized population of the US. The NHIS is an ongoing, nationally representative, household, in-person survey conducted by the National Center for Health Statistics, Centers for Disease Control and Prevention (http://www.cdc.gov/nchs/nhis). To facilitate stratification by ethnic group, we combined the three survey years (NHIS 2000, 2003 and 2005) containing detailed data on colorectal cancer screening procedures. For the purpose of this study, we analyzed all adults 50 years of age and older without a prior history of colon or rectal cancer and who self-reported being Hispanic/Latino. A current feature of the NHIS sampling design is the oversampling of Latinos, permitting analyses of Mexicans, who constitute the single largest Latino ethnic group in the US, followed by Puerto Ricans and Cubans ${ }^{34}$. Thus, analyses were conducted separately for Mexican $(\mathrm{N}=2,304)$, Puerto-Rican $(\mathrm{N}=503)$ and
Cuban ( $\mathrm{n}=484)$ adults to examine subgroup-specific relationships between acculturation and CRC screening.

\section{Dependent Variables}

Three CRC screening outcomes, available in the NHIS, were investigated: (1) whether individuals had undergone FOBT in the past year, (2) whether individuals had received a sigmoidoscopy in the last 5 years or colonoscopy in the last 10 years and (3) whether individuals had received any up-to-date CRC screening (or met the criterion for no. 1 or 2). These CRC screening criteria were based on clinical guidelines that were in place prior to the study period ${ }^{35}$.

\section{Independent Variables}

Acculturation was operationalized with two discrete variables: (1) nativity-whether the respondent was born outside the 50 US states or not (hereafter referred to as mainland US and (2) language preference-whether the respondent conducted the interview in English or not. Language has been shown to be a powerful indicator of ethnic identity, and nativity has been identified as a key objective measure of acculturation ${ }^{36-40}$. Nativity reflects exposure to US culture, and studies have shown that longer exposure to competing values of the larger society are expected to diminish the influence of values unique to an ethnic culture ${ }^{36}$.

We also analyze key covariates, including demographic variables (age, sex) and socio-economic factors known as predisposing variables (income status, education), enabling factors or those related to health-care access (type of insurance and usual source of care) and health-care need factors (number of chronic diseases), which is a count variable ranging from 0 to 6 , of whether the respondent reported having diabetes, hypertension, ulcer, arthritis, any cardiovascular disease and any respiratory illness.

\section{Statistical Analyses}

We examined bivariate and adjusted relationships between acculturation measures and all three CRC screening outcomes. We used chi-square tests to examine unadjusted associations between nativity and language of interview and the three outcomes. Using logistic regression, models containing nativity, language of interview and all covariates were estimated to assess the independent contributions of each on the three outcomes. All analyses were adjusted for complex survey design using SAS (version 9.1.3) and SUDAAN ${ }^{41}$.

\section{RESULTS}

\section{Sample Characteristics}

Table 1 presents characteristics of adults who met the study inclusion criteria (non-Hispanic Black and White adults are presented for comparative purposes). Overall, the majority of Mexicans and Puerto Ricans conducted the interview in English (approximately 60\%), while the majority of Cubans conducted the interview in Spanish or a combination of Spanish and English (75\%). The majority of Mexicans were born in the US (56\%). In contrast, the majority of Puerto Ricans and Cubans were born outside the mainland US, $(79 \%$ 
Table 1. Respondent Characteristics (\%) by Racial and Ethnic Group*, NHIS† 2000, 2003, 2005

\begin{tabular}{|c|c|c|c|c|c|c|}
\hline & Mexico $N=2,304$ & Puerto Rico $\mathrm{N}=503$ & Cuba $\mathrm{N}=484$ & Black $N=4,803$ & White $\mathrm{N}=\mathbf{2 8 , 3 0 6}$ & Total $\mathrm{N}=38,347$ \\
\hline \multicolumn{7}{|l|}{ Language of interview } \\
\hline English only & 57.9 & 60.3 & 25.0 & $\mathrm{n} / \mathrm{a}$ & $\mathrm{n} / \mathrm{a}$ & $\mathrm{n} / \mathrm{a}$ \\
\hline Spanish or Spanish/English & 42.1 & 39.7 & 75.0 & $\mathrm{n} / \mathrm{a}$ & $\mathrm{n} / \mathrm{a}$ & $\mathrm{n} / \mathrm{a}$ \\
\hline \multicolumn{7}{|l|}{ US nativity } \\
\hline Born in the US & 55.5 & 21.0 & 4.8 & $\mathrm{n} / \mathrm{a}$ & $\mathrm{n} / \mathrm{a}$ & $\mathrm{n} / \mathrm{a}$ \\
\hline Born outside the US & 44.5 & 79.0 & 95.2 & $\mathrm{n} / \mathrm{a}$ & $\mathrm{n} / \mathrm{a}$ & $\mathrm{n} / \mathrm{a}$ \\
\hline \multicolumn{7}{|l|}{ Family poverty level (FPL) } \\
\hline Missing & 31.2 & 23.5 & 24.6 & 29.0 & 29.8 & 29.6 \\
\hline $100 \% \mathrm{FPL}$ & 18.8 & 20.8 & 16.3 & 18.0 & 6.1 & 8.1 \\
\hline $100-124 \% \mathrm{FPL}$ & 6.7 & 6.8 & 6.9 & 6.6 & 3.4 & 4.0 \\
\hline $125-199 \%$ FPL & 13.6 & 14.9 & 17.5 & 12.5 & 10.6 & 10.9 \\
\hline 200-399\% FPL & 17.9 & 20.2 & 17.9 & 18.3 & 21.6 & 21.1 \\
\hline$\geq 400 \% \mathrm{FPL}$ & 11.9 & 13.8 & 16.9 & 15.5 & 28.5 & 26.4 \\
\hline \multicolumn{7}{|l|}{ Educational level } \\
\hline Less than high school & 58.9 & 50.1 & 46.5 & 36.4 & 17.2 & 21.2 \\
\hline High school/GED $\ddagger$ & 20.1 & 25.3 & 19.2 & 28.2 & 33.5 & 32.0 \\
\hline Some college & 14.4 & 16.4 & 14.3 & 22.3 & 25.2 & 24.2 \\
\hline College graduate or higher & 6.6 & 8.3 & 20.0 & 13.1 & 24.1 & 22.5 \\
\hline \multicolumn{7}{|l|}{ Type of insurance $\S$} \\
\hline Private & 21.5 & 21.2 & 16.6 & 23.2 & 26.8 & 26.1 \\
\hline Medicare & 36.2 & 44.2 & 51.7 & 43.1 & 49.3 & 47.6 \\
\hline Medicaid & 5.6 & 12.7 & 7.1 & 6.6 & 1.6 & 2.5 \\
\hline Other public & 14.2 & 13.0 & 11.6 & 17.0 & 17.0 & 16.9 \\
\hline Uninsured & 22.5 & 8.9 & 12.9 & 10.0 & 5.4 & 6.9 \\
\hline Has usual source of care & 54.6 & 60.3 & 59.0 & 59.9 & 60.9 & 60.3 \\
\hline
\end{tabular}

*Adults 50 years of age and older, never diagnosed with colorectal cancer

$\dagger$ NHIS = National Health Interview Survey

$\ddagger G E D=$ General equivalency diploma

$\S$ Adults 65 years old and over who reported Medicare and Medicaid were assigned to the Medicare category and those who reported Medicare and Private were assigned to the Private insurance category

Table 2. CRC Screening Outcomes by Nativity and Language of Interview*: NHIS 2000, 2003, 2005†

\begin{tabular}{|c|c|c|c|c|}
\hline & FOBT‡ in the past year & Up-to-date endoscopy $\S$ & Any up-to-date CRC screening & Total sample size \\
\hline Mexico & 10.9 & 23.6 & 29.0 & 1,749 \\
\hline \multicolumn{5}{|l|}{ Nativity } \\
\hline Born in the US & $13.3 \|$ & $28.1 \|$ & $35.4 \|$ & 1,031 \\
\hline Born outside the US & 7.3 & 16.7 & 21.4 & 718 \\
\hline \multicolumn{5}{|l|}{ Language of interview } \\
\hline English only & 13.1 I & 27.4 II & 35.1 वा & 1,031 \\
\hline Spanish/Spanish and English & 7.2 & 17.1 & 21.1 & 718 \\
\hline Puerto Rico & 13.9 & 28.5 & 35.5 & 456 \\
\hline \multicolumn{5}{|l|}{ Nativity } \\
\hline Born in the US & $6.0 \|$ & 28.6 & $31.3 \|$ & 88 \\
\hline Born outside the US & 15.9 & 28.5 & 36.5 & 368 \\
\hline \multicolumn{5}{|l|}{ Language of interview } \\
\hline English only & 16.3 II & 26.8 वा & 36.5 II & 263 \\
\hline Spanish/Spanish and English & 10.3 & 30.9 & 33.8 & 193 \\
\hline Cuba & 11.2 & 30.6 & 36.8 & 418 \\
\hline \multicolumn{5}{|l|}{ Nativity } \\
\hline Born in the US & $8.6 \|$ & $16.0 \|$ & $24.6 \|$ & 13 \\
\hline Born outside the US & 11.3 & 31.3 & 37.4 & 405 \\
\hline \multicolumn{5}{|l|}{ Language of interview } \\
\hline English only & 7.2 II & 28.7 वा & 33.6 वा & 78 \\
\hline Spanish/Spanish and English & 12.7 & 31.7 & 38.3 & 340 \\
\hline Non-Hispanic Black & 14.6 & 29.3 & 36.2 & 4,290 \\
\hline Non-Hispanic White & 16.8 & 38.3 & 45.3 & 26,629 \\
\hline
\end{tabular}

*Adults 50 years of age and older, never diagnosed with colorectal cancer

$\dagger$ NHIS = National Health Interview Survey

$\ddagger F O B T=$ Fecal occult blood test

$\S U$-to-date: flexible sigmoidoscopy in past 5 years or colonoscopy in past 10 years

$\| X^{2} p<0.0001$ comparing US and foreign-born categories

$\mathrm{II} \mathrm{X}^{2} p<0.0001$ comparing English language interview and Spanish/bilingual categories 
and 95\% respectively). Socioeconomic and health-care factors varied by national origin and racial and ethnic groups. For example, the percentages of college graduates ranged from $7 \%$ and $8 \%$, respectively, among Mexican and Puerto-Rican adults to $20 \%$ among Cubans. Finally, the percentage of uninsured ranged from 9\% among Puerto-Rican adults to 23\% among Mexican adults.

\section{Rates of CRC Screening According to Nativity and Language of Interview}

The rate of any up-to-date screening ranged from 29\% among Mexicans to $37 \%$ among Cubans, and the rate of up-to-date endoscopy ranged from 24\% among Mexicans and 31\% among Cubans (Table 2). There was less variation in FOBT in the past year according to ethnic group (Table 2).

There was wide variation by nativity and language of interview for all three CRC screening outcomes across ethnic groups. Among Mexicans, US-born adults and those who had English language interviews had significantly higher rates of all CRC screening outcomes measured compared to those born outside the US and who did not interview in English. In contrast, among Puerto-Rican and Cuban adults, rates for all
CRC screening outcomes were higher in foreign-born adults relative to their US-born counterparts (with the exception of endoscopy among Puerto Ricans where the rates were similar). Further, compared to Cubans who had a Spanish/bilingual interview, those who had an English language interview had lower rates of all CRC screening outcomes; this was also true for FOBT use among Puerto Ricans.

\section{Adjusted Analyses for Any Up-to-date Screening, FOBT in the Past Year, Up-to-date Endoscopy}

After adjusting for all covariates in the model, including demographic variables (age, sex), predisposing (income, education), enabling (insurance and usual source of care) and need (number of chronic conditions) factors central to the health service utilization model, there are significant independent associations between nativity and any up-to-date CRC screening (Table 3). For example, Mexicans who were born in the US had higher odds of reporting any up-to-date screening compared to those born outside the US (OR=1.4; 95\% CI: $1.0-$ 1.9). It is also notable that for Mexicans, family poverty level, education, usual source of care and number of chronic conditions all had independent associations with CRC screening.

Table 3. Adjusted Odds Ratios $(95 \% \mathrm{Cl})$ for Any Up-To-Date Colorectal Cancer Screening*: NHIS 2000, 2003, 2005†

\begin{tabular}{|c|c|c|c|}
\hline & Mexicans $\mathrm{N}=2,194$ & Puerto Ricans $N=483$ & Cubans $\neq N=409$ \\
\hline \multicolumn{4}{|l|}{ Nativity } \\
\hline Born in the US & $1.4(1.0,1.9) \S$ & $0.7(0.4,1.2)$ & $0.4(0.1,1.7)$ \\
\hline Born outside the US & 1.0 & 1.0 & 1.0 \\
\hline \multicolumn{4}{|l|}{ Language of interview } \\
\hline English only & $1.4(1.0,2.0)$ & $1.3(0.8,2.3)$ & $1.1(0.6,2.0)$ \\
\hline Spanish or Spanish/English & 1.0 & 1.0 & 1.0 \\
\hline \multicolumn{4}{|l|}{ Family poverty level (FPL) } \\
\hline Missing & $1.1(0.7,1.6)$ & $0.6(0.3,1.4)$ & $1.2(0.6,2.5)$ \\
\hline$\geq 400 \% \mathrm{FPL}$ & $2.1(1.3,3.4)$ & $1.7(0.7,4.1)$ & $2.8(1.3,6.0)$ \\
\hline $200-399 \% \mathrm{FPL}$ & $1.5(1.0,2.1)$ & $1.3(0.6,3.0)$ & $1.3(0.6,2.7)$ \\
\hline 100-199\% FPL & $1.3(0.9,1.8)$ & $0.6(0.3,1.2)$ & $1.6(1.0,2.8)$ \\
\hline$<100 \% \mathrm{FPL}$ & 1.0 & 1.0 & 1.0 \\
\hline \multicolumn{4}{|l|}{ Educational level } \\
\hline College graduate or higher & $2.4(1.4,3.9)$ & - & $0.8(0.5,1.5)$ \\
\hline Some college & $2.0(1.4,2.9)$ & $2.9(1.6,5.5) \|$ & $1.2(0.6,2.3)$ \\
\hline High school/GEDI & $1.4(1.0,2.0)$ & $1.6(0.9,2.6)$ & $0.7(0.3,1.3)$ \\
\hline Less than high school & 1.0 & 1.0 & 1.0 \\
\hline \multicolumn{4}{|l|}{ Type of insurance } \\
\hline Private & $1.2(0.7,1.9)$ & $3.1(1.1,8.6)$ & $0.5(0.2,1.3)$ \\
\hline Medicare & $1.5(0.9,2.6)$ & $3.5(1.3,9.3)$ & $1.9(0.8,4.6)$ \\
\hline Medicaid/other public & $1.4(0.8,2.2)$ & $3.0(1.2,7.9)$ & 1.0 \\
\hline Uninsured & 1.0 & 1.0 & \\
\hline \multicolumn{4}{|l|}{ Usual place of care } \\
\hline Has one or more place of care & $2.3(1.4,3.5)$ & $\mathrm{n} / \mathrm{a}$ & $\mathrm{n} / \mathrm{a}$ \\
\hline No usual place of care & 1.0 & $\mathrm{n} / \mathrm{a}$ & $\mathrm{n} / \mathrm{a}$ \\
\hline \multicolumn{4}{|l|}{ Sex } \\
\hline Male & $1.0(0.8,1.2)$ & $1.6(1.0,2.5) \S$ & $0.8(0.5,1.5)$ \\
\hline Female & 1.0 & 1.0 & 1.0 \\
\hline Age (continuous) no. & $1.1(1.0,1.2)$ & $1.1(1.0,1.3)$ & $1.1(1.0,1.2)$ \\
\hline $\begin{array}{l}\text { Number of chronic conditions } \\
\text { (continuous) }\end{array}$ & $1.3(1.2,1.4)$ & $1.4(1.2,1.6)$ & $1.6(1.3,2.0)$ \\
\hline
\end{tabular}

*Adults 50 years of age and older, never diagnosed with colorectal cancer; adjusted for survey year

$\uparrow$ NHIS = National Health Interview Survey

$\ddagger$ Limited to insured Cubans because of collinearity with nativity and small number of uninsured

\$95\% CI does not contain 1.0

|| Combines some college and higher

II GED = General equivalency diploma

\#Age variable is age/5: interpret magnitude in terms of every 5 years

$n / a=$ Not applicable; usual source of care not included because majority reported having one or more usual source of care (with too few cases reporting no usual source of care) 
In contrast, nativity and language of interview were not significant correlates of any up-to-date CRC screening among Puerto Ricans and Cubans (Table 3). Higher family income and a greater number of chronic conditions were significantly associated with a higher likelihood of any up-to-date CRC screening among Cubans. Insurance coverage, higher educational levels, male gender, and greater number of chronic conditions were also positively related to any up-to-date screening among Puerto Ricans only.

Interestingly, for FOBT, the study's acculturation measures were only significant among Puerto Ricans (Table 4). Puerto Ricans who were born in the mainland US were less likely to report receiving an FOBT in the past year compared to those born outside the US. In contrast, Puerto Ricans who interviewed in English were more likely to report FOBT in the past year than those who interviewed in Spanish. It is notable that higher educational level and a greater number of chronic conditions were also significant and positively related to having received an FOBT in the past year among Puerto Ricans. Among Mexicans, having a usual source of care and a greater number of chronic conditions were significant and positive correlates of FOBT in the past year. Among Cubans, male gender was the only significant, positive correlate of the outcome.
Nativity mattered in the receipt of up-to-date endoscopy only for Mexicans (Table 5). For Mexicans, those who were born in the US were more likely to report an up-to-date endoscopy than those born outside (1.5; CI: 1.1-2.2). Additionally, higher family incomes, higher educational levels, having a usual source of care and a greater number of chronic conditions were significant, positive correlates of up-to-date endoscopy among Mexicans. Higher family incomes, higher educational levels, male gender and a greater number of chronic conditions were significant, positive correlates among Puerto Ricans; higher family incomes, age and a greater number of chronic conditions were significant positive correlates among Cubans.

\section{DISCUSSION}

This study demonstrates that acculturation, inferred by nativity and language preference, is a significant determinant of CRC screening utilization in a nationally representative sample of older Latino adults even when taking into account a variety of health service utilization factors. For Mexican adults, US nativity was positively associated with any up-to-date screening or an up-to-date endoscopic procedure. The findings

Table 4. Adjusted Odds Ratios $(95 \% \mathrm{Cl})$ for Receipt of FOBT *in Past Yeart: NHIS 2000, 2003, 2005‡

\begin{tabular}{|c|c|c|c|}
\hline & Mexicans $\mathrm{N}=2,194$ & Puerto-Ricans $\mathrm{N}=483$ & Cubans $\S N=409$ \\
\hline \multicolumn{4}{|l|}{ Nativity } \\
\hline Born in the US & $1.3(0.9,2.0)$ & $0.3(0.2,0.7)$ & $1.5(0.2,13.8)$ \\
\hline Born outside the US & 1.0 & 1.0 & 1.0 \\
\hline \multicolumn{4}{|l|}{ Language of interview } \\
\hline English only & $1.3(0.9,1.9)$ & $2.5(1.1,5.4)$ & $0.7(0.2,2.0)$ \\
\hline Spanish or Spanish/English & 1.0 & 1.0 & 1.0 \\
\hline \multicolumn{4}{|l|}{ Family poverty level (FPL) } \\
\hline Missing & $1.3(0.7,2.3)$ & $0.5(0.2,1.7)$ & $0.8(0.3,2.5)$ \\
\hline$\geq 400 \% \mathrm{FPL}$ & $1.9(0.9,3.6)$ & $0.5(0.2,1.7)$ & $0.4(0.1,1.9)$ \\
\hline 200-399\% FPL & $1.7(0.9,3.3)$ & $1.4(0.6,3.3)$ & $0.7(0.2,2.1)$ \\
\hline 100-199\% FPL & $1.1(0.6,1.9)$ & $0.5(0.2,1.4)$ & $1.4(0.7,2.9)$ \\
\hline$<100 \% \mathrm{FPL}$ & 1.0 & 1.0 & 1.0 \\
\hline \multicolumn{4}{|l|}{ Educational level } \\
\hline College graduate or higher & $1.3(0.6,3.0)$ & $3.7(1.6,8.8) \mathrm{qL}$ & $1.2(0.5,2.5)$ \\
\hline Some college & $1.5(0.9,2.4)$ & & $0.8(0.3,2.4)$ \\
\hline High school/GED\| & $1.3(0.8,2.2)$ & $2.7(1.4,5.4)$ & $0.3(0.1,0.9)$ \\
\hline Less than high school & 1.0 & 1.0 & 1.0 \\
\hline \multicolumn{4}{|l|}{ Type of insurance } \\
\hline Private & $1.3(0.7,2.5)$ & $3.2(0.6,17.4)$ & $0.6(0.2,1.8)$ \\
\hline Medicare & $1.2(0.5,2.7)$ & $2.9(0.4,20.0)$ & $1.1(0.5,2.4)$ \\
\hline Medicaid/other public & $1.1(0.6,2.2)$ & $4.3(0.8,24.6)$ & 1.0 \\
\hline Uninsured & 1.0 & 1.0 & \\
\hline \multicolumn{4}{|l|}{ Usual place of care } \\
\hline Has one or more place of care & $3.3(1.1,9.0)$ & $\mathrm{n} / \mathrm{a}$ & $\mathrm{n} / \mathrm{a}$ \\
\hline No usual place of care & 1.0 & $\mathrm{n} / \mathrm{a}$ & $\mathrm{n} / \mathrm{a}$ \\
\hline \multicolumn{4}{|l|}{ Sex } \\
\hline Male & $1.2(0.8,1.6)$ & $1.0(0.5,2.0)$ & $2.4(1.3,4.6)$ \\
\hline Female & 1.0 & 1.0 & 1.0 \\
\hline Age (continuous)no. & $1.1(1.0,1.3)$ & $1.2(1.0,1.4)$ & $0.9(0.7,1.1)$ \\
\hline Number of chronic conditions (continuous) & $1.3(1.1,1.5)$ & $1.4(1.0,1.8) * *$ & $1.3(0.9,1.9)$ \\
\hline
\end{tabular}

*FOBT = Fecal occult blood test

$\dagger$ Adults 50 years of age and older, never diagnosed with colorectal cancer; adjusted for survey year

$\ddagger$ NHIS = National Health Interview Survey

$\S$ Limited to insured Cubans because of collinearity with nativity and small number of uninsured

$\| G E D=$ General equivalency diploma

IICombines some college and higher

\#Age variable is age/5: interpret magnitude in terms of every 5 years

** $95 \%$ CI does not contain 1.0

$n / a=$ Not applicable; usual source of care not included because majority reported having one or more usual source of care (with too few cases reporting no usual source of care) 
Table 5. Adjusted Odds Ratios $(95 \% \mathrm{Cl}$ ) for Up-To-Date Endoscopy*t: NHIS 2000, 2003, 2005 †

\begin{tabular}{|c|c|c|c|}
\hline & Mexicans $\mathrm{N}=2,194$ & Puerto Ricans $N=483$ & Cubans $\S \mathrm{N}=409$ \\
\hline \multicolumn{4}{|l|}{ Nativity } \\
\hline Born in the US & $1.5(1.1,2.2)$ & $0.9(0.5,1.7)$ & $0.2(0.1,1.1)$ \\
\hline Born outside the US & 1.0 & 1.0 & 1.0 \\
\hline \multicolumn{4}{|l|}{ Language of interview } \\
\hline English only & $1.3(0.9,1.9)$ & $0.7(0.4,1.5)$ & $1.2(0.6,2.2)$ \\
\hline Spanish or Spanish/English & 1.0 & 1.0 & 1.0 \\
\hline \multicolumn{4}{|l|}{ Family poverty level } \\
\hline Missing & $1.0(0.7,1.5)$ & $0.7(0.3,1.7)$ & $1.1(0.5,2.3)$ \\
\hline$\geq 400 \% \mathrm{FPL}$ & $2.1(1.3,3.4)$ & $2.7(1.0,7.0) \|$ & $3.5(1.7,7.3)$ \\
\hline $200-399 \% \mathrm{FPL}$ & $1.2(0.8,1.9)$ & $1.5(0.6,3.7)$ & $1.2(0.6,2.5)$ \\
\hline $100-199 \% \mathrm{FPL}$ & $1.4(1.0,2.1)$ & $0.7(0.3,1.3)$ & $1.5(0.8,2.8)$ \\
\hline$<100 \% \mathrm{FPL}$ & 1.0 & 1.0 & 1.0 \\
\hline \multicolumn{4}{|l|}{ Educational level } \\
\hline College graduate or higher & $2.5(1.5,4.0)$ & $2.4(1.2,4.6) \#$ & $0.7(0.4,1.3)$ \\
\hline Some college & $2.3(1.6,3.3)$ & & $1.0(0.5,2.0)$ \\
\hline High school/GED\| & $1.5(1.0,2.2) \mathrm{TI}$ & $1.4(0.8,2.6)$ & $0.7(0.4,1.4)$ \\
\hline Less than high school & 1.0 & 1.0 & 1.0 \\
\hline \multicolumn{4}{|l|}{ Type of insurance } \\
\hline Private & $1.2(0.7,2.1)$ & $2.2(0.7,6.8)$ & $0.7(0.3,1.7)$ \\
\hline Medicare & $1.8(1.0,3.1)$ & $3.4(1.2,10.0)$ & $2.5(1.0,6.4)$ \\
\hline Medicaid/other public & $1.5(0.9,2.5)$ & $2.2(0.7,6.8)$ & 1.0 \\
\hline Uninsured & 1.0 & 1.0 & \\
\hline \multicolumn{4}{|l|}{ Usual place of care } \\
\hline Has one or more place of care & $2.1(1.2,3.5)$ & $\mathrm{n} / \mathrm{a}$ & $\mathrm{n} / \mathrm{a}$ \\
\hline No usual place of care & 1.0 & $\mathrm{n} / \mathrm{a}$ & $\mathrm{n} / \mathrm{a}$ \\
\hline \multicolumn{4}{|l|}{ Sex } \\
\hline Male & $0.9(0.7,1.2)$ & $2.1(1.3,3.6)$ & $0.8(0.4,1.6)$ \\
\hline Female & 1.0 & 1.0 & 1.0 \\
\hline Age (continuous)** & $1.1(1.0,1.2)$ & $1.1(0.9,1.2)$ & $1.1(1.0,1.2) \mathrm{II}$ \\
\hline $\begin{array}{l}\text { Number of chronic conditions } \\
\text { (continuous) }\end{array}$ & $1.3(1.1,1.4)$ & $1.3(1.1,1.6)$ & $1.6(1.3,1.9)$ \\
\hline
\end{tabular}

*Up-to-date endoscopy = flexible sigmoidoscopy in the past 5 years or colonoscopy in the past 10 years

$\dagger$ Adults 50 years of age and older, never diagnosed with colorectal cancer; adjusted for survey year

$\ddagger$ NHIS = National Health Interview Survey

$\S$ Limited to insured Cubans because of collinearity with nativity and small number of uninsured

$\| G E D=$ General equivalency diploma

II95\% CI does not contain 1.0

\#Combines some college and higher

${ }^{* *}$ Age variable is age/5: interpret magnitude in terms of every 5 years

$n / a=$ Usual source of care not included because majority reported having one or more usual source of care (with too few cases reporting no usual source of care)

among Puerto Ricans were more complex with evidence of both positive and negative associations with the acculturation measures used in this study. Puerto Ricans who had an English language interview were more likely to receive FOBT in the past year relative to those who had a Spanish/bilingual interview; in contrast, Puerto Ricans who were born in the mainland US were less likely to receive FOBT in the past year relative to those born in Puerto Rico. There were no significant associations between acculturation and CRC screening among Cubans in adjusted analyses.

Enabling factors (insurance type and usual source of care) were also significant determinants of CRC screening for almost all ethnic groups and all CRC screening modalities. Further, for the more expensive screening modalities (e.g., colonoscopy or sigmoidoscopy), the conventional predisposing factors (income status and educational level, which can also be conceptualized as enabling factors) remained robust determinants. For FOBT, the less expensive test, however, predisposing factors were not significant determinants. The only exception to these findings was FOBT utilization among Puerto Ricans where, in addition to the acculturation measures and chronic conditions, educational level was a significant determinant.
These results are in contrast to those in an earlier study among Latinos that found an insignificant association between acculturation and CRC screening ${ }^{13}$. However, Shah et al. (2006) analyzed up-to-date screening among Latinos as an aggregate group, while our study disaggregated analyses by Latino national origin and CRC testing modality. Thus, aggregating ethnic groups and grouping CRC testing modalities together may lead to missed opportunities in identifying important predictors of CRC screening.

Our findings highlight the diversity of Latinos in the US by suggesting differential relationships between acculturation and CRC screening by national origin. Among Mexicans in our study, we observed a positive relationship: US nativity was associated with a higher likelihood of endoscopy and overall up-to-date CRC screening. Among Puerto Ricans, we observed the opposite relationship: US nativity was associated with a lower likelihood of FOBT use. These findings are consistent with evidence of differential associations by ethnic group between acculturation and preventive behaviors ${ }^{20}$. Allen et al. (2007) find overall improvement of preventive behaviors among Asian teens from one generation to the next and steady or worsening preventive behaviors among Latino teens ${ }^{20}$. These differential associations are also supported by contemporary 
theories of assimilation, which characterize heterogeneous paths of social mobility from one generation to the next ${ }^{28}$, leading to better or worse preventive behaviors.

The findings among Mexicans suggest US natives, relative to their Mexican origin counterparts, may be more likely to possess certain social norms or skills that encourage or facilitate cancer screening utilization. For example, because of their greater exposure to the US culture and medical system, US-born Mexicans may be more comfortable at discussing their health concerns with providers, be more skilled at navigating the health-care system or have more exposure to social circles that support positive screening behaviors when compared to their Mexican origin counterparts $^{11,17,24,42-44}$. Older Mexican adults born in Mexico, relative to those born in the US, may also be more likely to mistrust the medical system, an interpretation consistent with a study of acculturation and breast cancer screening, which found that less acculturated Latina women were more likely to be suspicious of their medical care and screened less frequently ${ }^{45}$.

In contrast to the older Mexican adults in our study, mainland US nativity has a negative association with FOBT use among Puerto Ricans. This finding is consistent with recent work comparing willingness to participate in cancer screening among San Juan and New York City (NYC) Puerto Ricans, which found higher rates among San Juan Puerto Ricans ${ }^{46}$. Claudio et al. ${ }^{46}$ explain that San Juan Puerto Ricans, relative to NYC Puerto Ricans, possibly have greater exposure to health information and services because of the strong presence of the pharmaceutical and health-care industry in Puerto Rico, which is also a possibility in this study. Further, consistent with studies finding a positive association between provider patient language concordance and health care, Puerto-Rican health-care providers, compared to US healthcare providers, may have been more effective at promoting FOBT use among their Puerto-Rican patients because of their shared culture and language $\mathrm{e}^{47-49}$.

Finally, an important finding of this study is the divergent relationships between nativity and language of interview and FOBT use among Puerto Ricans. Because Puerto Rico is a US territory, mainland US nativity and English language preference may reflect different constructs than they do in other ethnic groups. While mainland US nativity may reflect exposure to imperfect social and health-care systems, English preference may reflect a process of acculturation that leads to a weakening of attitudes that foster feelings of fear, embarrassment or invulnerability discussed in previous studies of Latinos and Puerto Ricans in particular ${ }^{50,51}$, and adoption of more positive beliefs about cancer screening.

We should note some important limitations. First, estimated rates of CRC screening were based on self-report. However, a prior study found good agreement between self-report data and medical records for sigmoidoscopy and colonoscopy $^{52}$. Second, we were not able to discern whether colonoscopies were performed for diagnostic or screening purposes, potentially overestimating CRC screening rates. Third, we were unable to examine physician recommendations for the test because NHIS does not collect these data. Physician recommendation data were collected in the 2000 and 2005 NHIS, but only for respondents who were never screened or who did not screen within the recommended timeframe.
US nativity and interview language were the only acculturation proxies available in all 3 years of NHIS. Although alone these measures may not capture the complexity of the acculturation process, US nativity offers an approximation of exposure to the dominant culture, while language measures have been shown to explain most of the variance in acculturation scales ${ }^{53}$. Further, both measures constitute a systematic approach to measuring acculturation ${ }^{12-19,31,39}$.

\section{CONCLUSION}

The results underscore the importance of targeting less acculturated individuals to promote CRC screening utilization in ethnically diverse settings. Clinicians, however, need to recognize the complexity of acculturation and treat common indicators, such as nativity and language as independent dimensions among their Latino patients.

Acknowledgement: Authors wish to thank Kathryn A. Phillips, $\mathrm{PhD}$, for her comments on an earlier draft of this paper. An earlier version of this paper was presented at the 60th Annual Scientific Meeting of The Gerontological Society of America on November 19, 2007. The research for this paper was supported by the National Cancer Institute (NCI) Diversity Supplement to RO1 CA101849 awarded to Dr. Afable-Munsuz. Dr. Ponce's time was supported by NCI/NIH KO7 100097.

Conflict of Interest: None disclosed.

Corresponding Author: Aimee Afable-Munsuz; PhD, Division of General Internal Medicine, Department of Medicine, University of California, 3333 California Street, Box 0856, San Francisco, CA 94143, USA (e-mail: aafable-munsuz@ucsf.edu).

\section{REFERENCES}

1. Jemal A, Siegel R, Ward E, et al. Cancer statistics, 2008. CA Cancer J Clin. 2008; 582: 71-96.

2. Levin B, Lieberman DA, McFarland B, et al. Screening and surveillance for the early detection of colorectal cancer and adenomatous polyps, 2008: a joint guideline from the American Cancer Society, the US Multi-Society Task Force on Colorectal Cancer, and the American College of Radiology. Gastroenterology. 2008; 1345: 1570-95.

3. Phillips KA, Liang SY, Ladabaum U, et al. Trends in colonoscopy for colorectal cancer screening. Med Care. 2007; 452: 160-7.

4. Ananthakrishnan AN, Schellhase KG, Sparapani RA, Laud PW, Neuner JM. Disparities in colon cancer screening in the Medicare population. Arch Intern Med. 2007; 1673: 258-64.

5. Shih YC, Zhao L, Elting LS. Does Medicare coverage of colonoscopy reduce racial/ethnic disparities in cancer screening among the elderly? Health Aff (Millwood). 2006; 254: 1153-62.

6. Liang SY, Phillips KA, Nagamine M, Ladabaum U, Haas JS. Rates and predictors of colorectal cancer screening. Prev Chronic Dis. 2006; 34: A117.

7. Swan J, Breen N, Coates RJ, Rimer BK, Lee NC. Progress in cancer screening practices in the United States: results from the 2000 National Health Interview Survey. Cancer. 2003; 976: 1528-40.

8. Goel MS, Wee CC, McCarthy EP, Davis RB, Ngo-Metzger Q, Phillips RS. Racial and ethnic disparities in cancer screening: the importance of foreign birth as a barrier to care. J Gen Intern Med. 2003; 1812: 1028-35.

9. Gany FM, Herrera AP, Avallone M, Changrani J. Attitudes, knowledge, and health-seeking behaviors of five immigrant minority communities in the prevention and screening of cancer: a focus group approach. Ethn Health. 2006; 111: 19-39.

10. Johnson CE, Mues KE, Mayne SL, Kiblawi AN. Cervical cancer screening among immigrants and ethnic minorities: a systematic review using the Health Belief Model. J Low Genit Tract Dis. 2008; 123: 232-41. 
11. Fernandez ME, Wippold R, Torres-Vigil I, Byrd T, Freeberg D, Bains $\mathbf{Y}$, Guajardo J, Coughlin SS, Vernon Sw. Colorectal cancer screening among Latinos from U.S. cities along the Texas-Mexico border. Cancer Causes Control. 2008; 192: 195-206. Epub 2007 Nov 24.

12. Shah M, Zhu K, Potter J. Hispanic acculturation and utilization of colorectal cancer screening in the United States. Cancer Detect Prev. 2006; 303: 306-12

13. Yepes-Rios M, Reimann JO, Talavera AC, Ruiz de Esparza A, Talavera GA. Colorectal cancer screening among Mexican Americans at a community clinic. Am J Prev Med. 2006; 303: 204-10.

14. Tang TS, Solomon LJ, McCracken LM. Barriers to fecal occult blood testing and sigmoidoscopy among older Chinese-American women. Cancer Pract. 2001; 96: 277-82.

15. Teng EJ, Friedman LC, Green CE. Determinants of colorectal cancer screening behavior among Chinese Americans. Psychooncology. 2006; 155: 374-81

16. Yip MP, Tu SP, Chun A, Yasui Y, Taylor VM. Participation in colorecta cancer screening among Chinese Americans. Asian Pac J Cancer Prev. 2006; 74: 645-50.

17. Honda K. Factors associated with colorectal cancer screening among the US urban Japanese population. Am J Public Health. 2004; 945: 815-22.

18. Maxwell AE, Bastani R, Warda US. Demographic predictors of cancer screening among Filipino and Korean immigrants in the United States. Am J Prev Med. 2000; 181: 62-8.

19. Ponce NA, Huh S, Bastani R. Do HMO market level factors lead to racial/ethnic disparities in colorectal cancer screening? A comparison between high-risk Asian and Pacific Islander Americans and high-risk whites. Med Care. 2005; 4311: 1101-8.

20. Allen ML, Elliott MN, Morales LS, Diamant AL, Hambarsoomian K, Schuster MA. Adolescent participation in preventive health behaviors, physical activity, and nutrition: differences across immigrant generations for Asians and Latinos compared with Whites. Am J Public Health. 2007; 972: 337-43

21. Zsembik BA, Fennell D. Ethnic variation in health and the determinants of health amond Latinos. Soc Sci Med. 2005; 61: 53-63.

22. Zambrana RE, Breen N, Fox SA, Gutierrez-Mohamed ML. Use of cancer screening practices by Hispanic women: analyses by subgroup. Prev Med. 1999; 296 Pt 1: 466-77.

23. O'Malley AS, Kerner J, Johnson AE, Mandelblatt J. Acculturation and breast cancer screening among Hispanic women in New York City. Am J Public Health. 1999; 892: 219-27.

24. Solis JM, Marks G, Garcia M, Shelton D. Acculturation, access to care, and use of preventive services by Hispanics: findings from HHANES 1982-84. Am J Public Health. 1990; 80Suppl: 11-9.

25. Abraido-Lanza AF, Chao MT, Gates CY. Acculturation and cancer screening among Latinas: results from the National Health Interview Survey. Ann Behav Med. 2005; 291: 22-8.

26. Gorin SS, Heck JE. Cancer screening among Latino subgroups in the United States. Prev Med. 2005; 405: 515-26.

27. Suarez L. Pap smear and mammogram screening in Mexican-American women: the effects of acculturation. Am J Public Health. 1994; 845: 742-6.

28. Portes A, Zhou M. The New Second Generation: Segmented Assimilation and Its Variants. The Annals of the American Academy. 1993;530 (November 1990)

29. Massey DS. Latinos, poverty, and the underclass: a new agenda for research. Hisp J Behav Sci. 1993; 154: 449-75.

30. Berry Jw. Acculturation and adaptation in a new society. International Migration. 30:69-85

31. Lara M, Gamboa C, Kahramanian MI, Morales LS, Bautista DE. Acculturation and Latino Health in the Unites States: A Review of the Literature and its Sociopolitical Context. Annu Rev Public Health. 2005; 26: 367-97.
32. Marin G, Sabogal F, Marin BV, Otero-Sabogal R, Perez-Stable EJ. Development of a Short Acculturation Scale for Hispanics. Hisp J Behav Sci. 1987; 92: 183-205.

33. Andersen RM. Revisiting the behavioral model and access to medical care: does it matter? J Health Soc Behav. 1995; 361: 1-10.

34. Ramirez, R. We the People: Hispanics in the United States. U.S. Census Bureau, Census 2000 Special Report, CENSR-18, December 2004.

35. Smith RA, von Eschenbach AC, Wender R, et al. American Cancer Society guidelines for the early detection of cancer: update of early detection guidelines for prostate, colorectal, and endometrial cancers. Also: update 2001-testing for early lung cancer detection. CA Cancer J Clin. 2001; 511: 38-75. quiz 77-80.

36. Bean FD, Swicegood G. Generation, female education and MexicanAmerican fertility. Soc Sci Q. 1982; 631: 131-44.

37. Giles H, Johnson P. Ethnolinguistic identity theory: A social psychological approach to language maintenance. Int J Soc Language. 1987; 68: 69-100.

38. Tong $\mathbf{Y}$, Hong $\mathbf{Y}$, Lee $\mathbf{S}$, et al. Language use as a carrier of social identity. Int J Intercultural Relations. 1999; 23: 281-96.

39. Afable-Munsuz A, Brindis CD. Acculturation and the sexual and reproductive health of Latino youth in the United States: a literature review. Perspect Sex Reprod Health. 2006; 384: 208-19.

40. Finch BK, Boardman JD, Bohdam K, Vega WA. Contextual effects of acculturation on perinatal substance exposure among immigrant and native-born latinas. Soc Sci Q. 2000; 811: 420-38.

41. SAS. Version 9.1.3. Cary, NC: SAS Institute; 2002-2003.

42. Ponce NA, Hays RD, Cunningham WE. Linguistic disparities in health care access and health status among older adults. J Gen Intern Med. 2006; 217: 786-91.

43. Guesada GM. Language and communication barriers for health delivery to a minority group. Soc Sci Med. 1976; 106: 323-7.

44. Diaz JA, Roberts MB, Goldman RE, Weitzen S, Eaton CB. Effect of language on colorectal cancer screening among Latinos and non-Latinos. Cancer Epidemiol Biomarkers Prev. 2008; 178: 2169-73.

45. Thompson HS, Valdimarsdottir HB, Winkel G, Jandorf L, Redd W. The group-based medical mistrust scale: psychometric properties and association with breast cancer screening. Prev Med. 2004; 382: 209-18.

46. Claudio C, Katz RV, Green BL, Kressin NR, Wang MQ, Russell SL Cancer screening participation: comparative willingness of San Juan Puerto Ricans versus New York City Puerto Ricans. J Natl Med Assoc. 2007; 995: 542-9.

47. Eamranond PP, Davis RB, Phillips RS, Wee CC. Patient-Physician language concordance and lifestyle counseling among Spanish-speaking patients. J Immigr Minor Health. 2009;19.

48. Ngo-Metzger Q, Sorkin DH, Phillips RS. Providing high-quality care for limited English proficient patients: the importance of language concordance and interpreter use. J Gen Intern Med. 2007; 22Suppl 2: 324-30.

49. Wilson E, Chen AH, Grumbach K, Wang F, Fernandez A. Effects of limited English proficiency and physician language on healthcare comprehension. J Gen Intern Med. 2005; 20: 800-6.

50. Goldman R. Barriers to intimate cancer screening testing among Dominican and Puerto Rican men. http://www.brown.edu/Research/ ICAP/Modules/Modl ensHealthy/TeachingMats/Handout3Hisponic Males.doc. Accessed Jan. 2009

51. Perez-Stable EJ, Otero-Sabogal R, Sabogal F, et al. Self-reported use of cancer screening tests among Latinos and Anglos in a prepaid health plan. Arch Intern Med. 1994; 154: 1073-81.

52. Hall HI, Van Den Eeden SK, Tolsma DD, et al. Testing for prostate and colorectal cancer: comparison of self-report and medical record audit Prev Med. 2004; 391: 27-35.

53. Marin G. Issues in the measurement of acculturation among HispanicsIn: Geisenger KF, ed. Psychological Testing of Hispanics. Washington, DC: American Psychological Association; 1992: 23-51. 